\title{
Fatores de risco para comportamentos autolesivos em adolescentes escolares
}

\section{Risk factors for self-harming behavior in schools}

\author{
1 Joyce Lopes Macedo joycelopes385@gmail.com \\ 2 Amanda Suellenn da Silva Santos Oliveira \\ ${ }^{3}$ Erica Rodrigues Reis \\ ${ }^{4}$ Magnólia de Jesus Sousa Magalhães Assunção
}

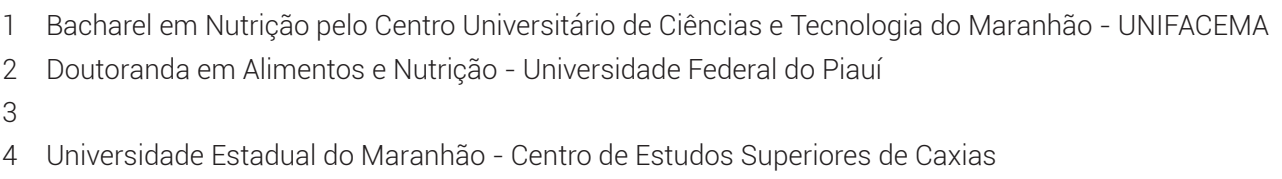

\section{Resumo}

O presente artigo tem por finalidade realizar uma busca bibliográfica sobre os fatores de risco para comportamentos autolesivos em adolescentes escolares. Trata-se de uma revisão de literatura do tipo integrativa. Para busca e seleção dos estudos, utilizou-se as seguintes bases de dados: Google acadêmico, Scielo e Pubmed, publicados entre os anos de 2006 a 2019, considerando artigos publicados em português e inglês. Em todos os estudos analisados, foi possível verificar a existência de diversos fatores associados à conduta autolesiva em escolares. Inúmeras pesquisas têm explorado alguns fatores que parecem estar relacionados ao comportamento autolesivo, aumentando o risco ou funcionando de forma protetora. Concluiu-se que é necessário a realização de mais estudos que abordem a temática, no intuito de compreender os principais fatores associados à prática de autolesão por adolescentes escolares, como forma de conscientização e implementação de estratégias voltadas à saúde do público-alvo.

\section{Palavras-chave}

Autolesão. Escolares. Adolescentes. Saúde mental.

\begin{abstract}
The purpose of this article is to conduct a bibliographic search on the risk factors for self-harming behavior in school adolescents. It is an integrative literature review. To search and select the studies, the following databases were used: Google Scholar, Scielo and Pubmed, published between the years 2006 to 2019, considering articles published in Portuguese and English. In all the studies analyzed, it was possible to verify the existence of several factors associated with self-harming conduct in schoolchildren. Numerous studies have explored factors that seem to be related to self-injurious behavior, increasing the risk or working in a protective way. It was concluded that it is necessary to carry out more studies that address the theme, in order to understand the main factors associated with the practice of self-harming by school adolescents, as a way of raising awareness and implementing strategies aimed at the health of the target audience.
\end{abstract}

\section{Keywords}

Self-harming. Schoolchildren. Adolescents. Mental health.

\section{Como você deve citar?}

MACEDO, Joyce Lopes et al. Fatores de risco para comportamentos autolesivos em adolescentes escolares. Cadernos UniFOA, Volta Redonda, n. 42, p. 75-79, abril, 2020. 


\section{INTRODUÇÃO}

A adolescência caracteriza-se por diversas alterações em níveis distintos, tanto físico como mental e social, com consequente aquisição de características e competências que a capacite para assumir os deveres do adulto e se integrar à sociedade. É, portanto, um período particularmente vulnerável, no qual existe uma potencialidade acentuada de mudança, mas também de desequilíbrio, em que os comportamentos autolesivos atingem proporções alarmantes (COSTA, 2012).

Autolesão se refere a um conjunto de comportamentos que resultam em dano intencional ao indivíduo, com conhecimento de que podem trazer algum grau de injúria física ou psicológica (NOCK, 2009). Em geral, classifica-se o comportamento autolesivo em dois grandes grupos: no primeiro, o ato de se lesionar é a intenção do comportamento, enquanto que, no segundo, caracteriza-se por ser o produto ou resultado de uma ação que não objetiva tal dano (NOCK, 2010).

Segundo Parks (2011), o primeiro incidente autolesivo é acidental ou impulsivo, quando a pessoa sente raiva, medo ou ansiedade de forma que não sabe como expressá-lo. A conduta autolesiva atua promovendo um senso momentâneo de alívio, às vezes, seguido de vergonha e culpa, até que fortes sentimentos surjam de novo, fazendo com que a pessoa se sinta emocionalmente sobrecarregada, levando-a a procurar alívio novamente em comportamentos autolesivos, que podem aumentar em frequência e grau.

Dessa forma, é necessário entender as causas que levam o adolescente à autolesão. Este estudo pode ser útil, ainda, para ajudar a explicar o engajamento em outros tipos de comportamentos prejudiciais, além de fomentar o debate em torno de questões para futuras pesquisas, principalmente, em nível nacional.

Diante do que foi exposto, o presente artigo teve por objetivo realizar uma revisão bibliográfica sobre os principais fatores de risco para comportamentos autolesivos em adolescentes escolares.

\section{MATERIAIS E MÉTODOS}

Este estudo caracteriza-se como uma revisão bibliográfica do tipo integrativa. Esse tipo de revisão consiste na construção de uma análise da literatura, importante para discussões sobre métodos e resultados de pesquisas. 0 propósito inicial é obter um profundo entendimento de um determinado fenômeno, baseando-se em estudos anteriores, de maneira sistemática e ordenada, contribuindo para o aprofundamento do conhecimento do tema investigado (MENDES; SILVEIRA; GALVÃO, 2008).

Para tanto, realizou-se a seguinte sequência de busca: $1^{\text {a }}$ etapa - identificação do tema e seleção da hipótese; $2^{\mathrm{a}}$ etapa - busca nas bases de dados eletrônicas; $3^{\mathrm{a}}$ etapa - definição das informações a serem extraídas dos estudos selecionados; $4^{a}$ etapa - estabelecimento de critérios para inclusão e exclusão de estudos; $5^{\mathrm{a}}$ etapa - interpretação dos resultados e apresentação da revisão/síntese do conhecimento.

Para a identificação do problema, formulou-se a seguinte pergunta norteadora do estudo: o que foi produzido na literatura sobre os fatores de risco para comportamentos autolesivos em adolescentes escolares?

A busca de dados foi realizada por meio de consulta a periódicos de referência na área e posterior leitura criteriosa dos títulos e dos resumos. 
Joyce Lopes Macedo I Amanda Suellenn da Silva Santos Oliveira Erica Rodrigues Reis I Magnólia de Jesus Sousa Magalhães Assunção

Como critérios de inclusão, foram utilizados artigos que respondiam à questão do estudo, que abordavam assuntos relacionados à temática, publicados no período de 2005 a 2019. Utilizou-se, para desenvolvimento do trabalho, as seguintes bases de dados: Google acadêmico, Scielo e Pubmed. Como critério de exclusão, os que não diziam respeito ao propósito deste estudo, que estavam repetidos nas diferentes bases de dados ou não apresentavam o texto completo na íntegra e com ano de publicação inferior a 2005.

Para a seleção dos artigos foram realizadas leituras previas através dos resumos, sendo escoIhidos os de maior relevância na literatura. Após leitura do material selecionado, agrupou-se por temas e as informações capturadas foram disponibilizadas em quadros, para posterior discussão.

\section{RESULTADOS E DISCUSSÃO}

Foram encontrados inicialmente 12.543 artigos. Em relação ao ano de publicação, os artigos eram do período de 2006 a 2019 (Fluxograma 1).

Fluxograma 1 - Processo de seleção do estudo.

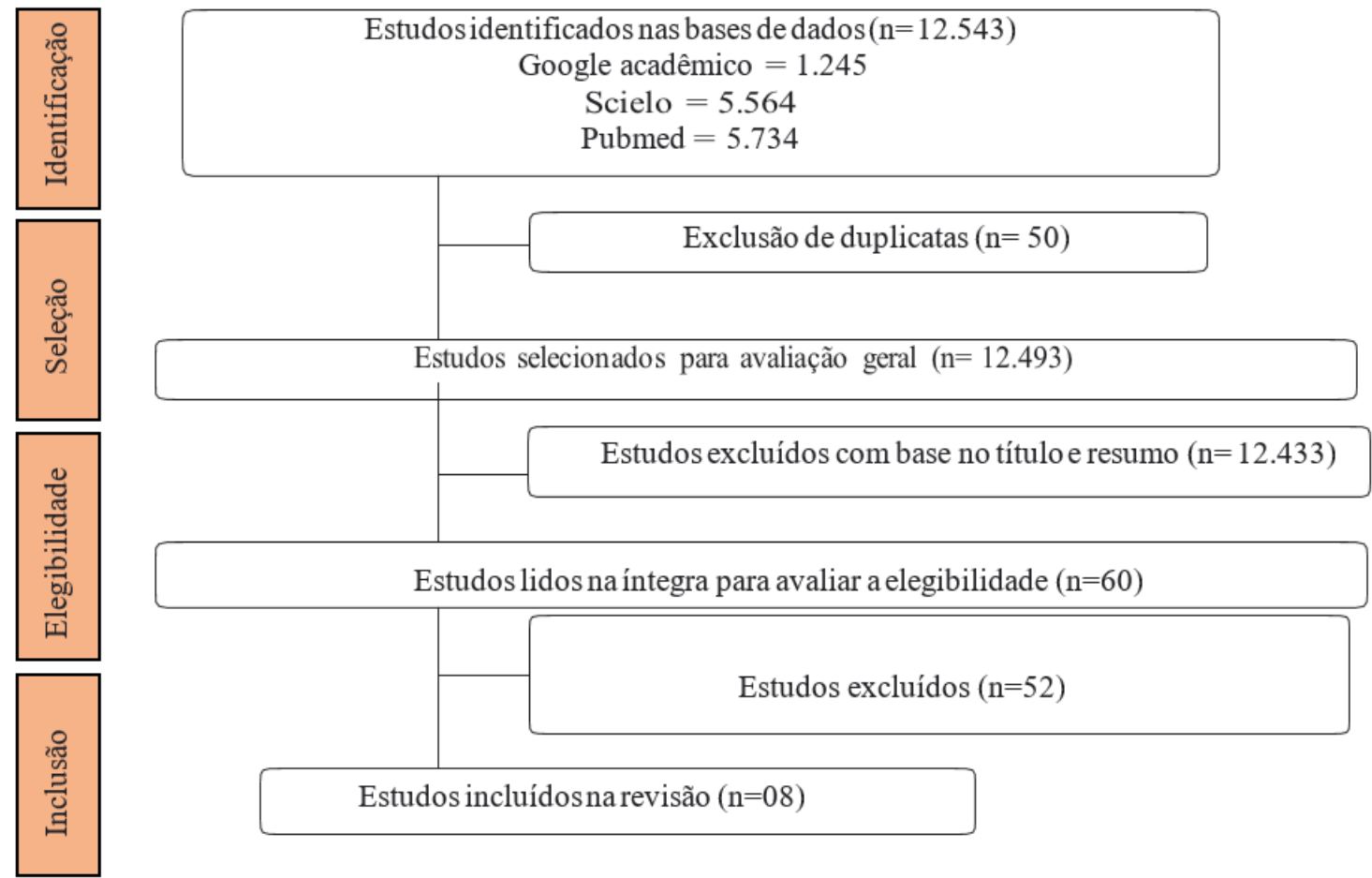

Fonte: autores, 2020

Pesquisas sobre comportamentos autolesivos têm explorado alguns fatores que parecem estar relacionados ao comportamento em questão, aumentando o risco ou funcionando de forma protetora.

Aspectos psicopatológicos são frequentemente apontadas como relacionadas ao comportamento autolesivo. Brickman et al. (2014) citam que quatro sintomas específicos do Transtorno de Personalidade Borderline são bastante associados com riscos para autolesionar-se: história prévia de pensamento ou comportamento suicida, comportamentos impulsivos recorrentes, autoimagem imprecisa e perturbações identitárias. 
Segundo Parks (2011), a conduta autolesiva é um comportamento muito observado também em pessoas diagnosticadas com autismo, em decorrência da baixa capacidade de comunicação desses indivíduos, funcionando, muitas vezes, como uma forma de expressão.

Fatores ambientais também são citados, envolvidos com a ocorrência do comportamento autolesivo, tais como eventos de vida e situações relacionadas a amigos e familiares, visto que exercem papel fundamental no desenvolvimento de crianças e adolescentes. Dessa forma, adolescentes que possuem uma relação parental inconsistente e insensível apresentam maior probabilidade de se engajarem em comportamentos que tragam danos a si próprios (KLONSKY; LEWIS, 2014).

A literatura também menciona que o período em que a influência dos pares aumenta, fato comum que caracteriza a transição para a adolescência, coincidindo com o surgimento de auto injúria nesse ciclo de vida (NOCK, 2009).

Alguns aspectos da relação com os pares parecem influenciar a ocorrência do comportamento, tais como problemas interpessoais (rejeição, conflitos) e dificuldades emocionais (CHAPMAN; GRATZ; BROWN, 2006). Além disso, ainda nesse período, no ciclo de vida até o início da idade adulta, a autoimagem negativa tem sido associada à vulnerabilidade para autolesão.

Muehlenkamp e Brausch (2012) relatam que fatores biológicos, como mudanças bioquímicas corporais, desempenham papel relevante para comportamentos autolesivos e podem ser resultantes de alguma ação específica como a ingestão de substâncias ou mesmo condições médicas. Esses fatores incluem insônia, fadiga, doenças ou anomalias da tireoide ou hormonais, síndrome pré-menstrual, ingestão de substâncias psicoativas e outros que possam aumentar a vulnerabilidade ao estresse e, consequentemente, o risco de engajar-se em comportamentos de autolesão.

Outro fator associado à conduta autolesiva são os vieses cognitivos. Compreender os tipos de pensamentos relacionados a esse comportamento pode ser importante para o entendimento das causas que levam ao engajamento (KLONSKY; LEWIS, 2014).

Pensamentos recorrentes em indivíduos que se lesionam são "só vou fazer um corte", "isso é demais para suportar", "eu mereço essa dor", "me cortar alivia mais do que qualquer outra coisa", "a vida é uma droga", "estou sozinho, não tenho amigos". Tais pensamentos influenciam a probabilidade de o comportamento aumentar ou diminuir a frequência e intensidade (WALSH, 2006). Atitudes mais favoráveis em relação à autolesão (perceber o comportamento como eficaz, por exemplo) têm sido associada à intenção de se envolver no comportamento de novo no futuro (O'CONNOR; ARMITAGE; GRAY, 2006).

\section{CONSIDERAÇOES FINAIS}

O presente artigo teve como centralidade a temática que trata sobre o comportamento autolesivo. De forma mais específica, buscou-se a resposta para a pergunta inicial: o que foi produzido na literatura sobre os fatores de risco para comportamentos autolesivos em adolescentes escolares?

Como forma de resposta à questão norteadora, realizou-se uma revisão integrativa da literatura, abrangendo artigos científicos, disponíveis nas bases de dados: Google acadêmico, Scielo e Pubmed. Descartou-se os estudos que não diziam respeito ao propósito deste estudo, que estavam repetidos nas diferentes bases de dados, não apresentavam o texto completo na íntegra e fora do recorte temporal previamente estabelecido. 
Joyce Lopes Macedo / Amanda Suellenn da Silva Santos Oliveira Erica Rodrigues Reis I Magnólia de Jesus Sousa Magalhães Assunção

A autolesão é uma realidade frequente na população adolescente. No presente estudo, observou-se como fatores de risco, aspectos psicopatológicos, fatores ambientais, influência dos pares, fatores biológicos e vieses cognitivos.

Considerando-se a alta prevalência do comportamento autolesivo na adolescência e suas consequências e que comportamentos ocorridos nessa faixa etária podem estender-se à vida adulta, estudar a autolesão em adolescentes fomenta estratégias de intervenção e prevenção mais eficazes.

Espera-se que o reconhecimento dessa situação possibilite o interesse de novos pesquisadores, para a realização de mais estudos com enfoque na temática abordada, no intuito de compreender os principais fatores associados à prática de autolesão por adolescentes, como forma de conscientização e implementação de estratégias voltadas à saúde do público-alvo.

\section{REFERÊNCIAS}

BRICKMAN, L. J.; AMMERMAN, B. A..; LOOK, A. E.; BERMAN, M. E.; MCCLOSKEY, M. S. The relationship between non-suicidal self-injury and borderline personality disorder symptoms in a college sample. Borderline Personality Disorder and Emotion Dysregulation, p. 1-14, 2014.

CHAPMAN, A. L.; GRATZ, K. L.; BROWN, M. Z. Solving the puzzle of self-harm: the experiential avoidance model. Behaviour Research and Therapy, v. 44, n. 3, p. 371-394, 2006.

COSTA, I. Adolescência: Ideação suicida, depressão, desesperança e memórias autobiográficas. Dissertação, Instituto Universitário das Ciências Psicológicas, Sociais e da Vida, 2012.

KLONSKY, E. D.; LEWIS, S. P. Assessment of Nonsuicidal Self-injury. New York: Oxford University Press, 2014.

MENDES, K. D. S.; SILVEIRA, R. C. C. P.; GALVÃO, C. M. Revisão integrativa: método de pesquisa para a incorporação de evidências na saúde e na enfermagem. Texto contexto-enferm, v. 17, n. 4, p. 754-758, 2008.

MUEHLENKAMP, J. J.; BRAUSCH, A. M. Body regard and non-suicidal self-injury in adolescents. Journal of Adolescence, v. 35, p. 1-9, 2012.

NOCK, M. K. Why do people hurt themselves? New insights into the nature and functions of self-injury. Current Directions in Psychological Science, v. 18, n. 2, p. 78-83, 2009.

NOCK, M. K. Self-injury. Annual Review of Clinical Psychology, v. 6, p. 339-363, 2010.

O'CONNOR, R. C., ARMITAGE, C. J., GRAY, L. (2006). The role of clinical and social cognitive variables in parasuicide. British Journal of Clinical Psychology, 45, 465-481

PARKS, P. J. Self-injury Disorder. California: Reference Point Press, 2011.

WALSH, B. W. Treating self-injury: a pratical guide. New York: Guilford Press, 2006. 